\section{5-5.5 SOCIAL CAPITAL AND HEALTH: PURELY A QUESTION OF CONTEXT?}

doi:10.1136/jech.2011.142976b.62

G Giordano, * H Ohlsson, M Lindström. Lund University Medical Faculty, Malmö, Skåne, Sweden

Introduction Despite association between "social capital" and health outcomes having been extensively researched, debate still surrounds which level of analysis is most appropriate to investigate this contextual phenomenon. Traditional contexts such as "neighbourhood" seem only to explain a very small amount ( 5\%) of total variance in individual health outcomes, often chosen more out of data availability than as representations of individuals' social interactions and networks. The aims of this United Kingdom study are twofold; to investigate the strength of association between social capital and health at individual- and aggregated-levels; and second, to determine which context (household vs neighbourhood) better explains variation in individual-level health.

Methods Applying multilevel analyses to British Household Panel Survey data ( $\mathrm{N}=10992)$, we estimated fixed and random effects between individual-, household- and small area-level social capital indicators and general health. We further compared the variance in health attributable to each level using intraclass correlations.

Results Association between social capital and health seems to depend on indicator type and context, with one fifth of individuallevel health variance found at the household-level. However, only individual-level variables appear to influence contextual-level variance. Conclusion Social capital research could be advanced by focusing on contexts based on extended social networks, not just geographic proximity of random individuals. Furthermore, decision makers must now appreciate that social capital interventions targeting health are unlikely to be cost-effective if directed solely at the "community" level, as only small variations in health are attributable to such a context.

\section{5-5.6 EVALUATION OF THE COMPRESSION EXPANSION AND DYNAMIC EQUILIBRIUM THEORIES USING WESTERN AUSTRALIAN LINKED HOSPITAL MORBIDITY AND MORTALITY DATA}

doi:10.1136/jech.2011.142976b.63

\footnotetext{
${ }^{1,2}$ R Moorin, ${ }^{*}$ D Holman. ${ }^{1}$ Curtin University, Western Australia, Australia; ${ }^{2}$ University of
} Western Australia, Western Australia, Australia

Introduction Three hypotheses have been advanced to predict changes in population health in countries experiencing low birth and death rates, and increasing expectation of life. Determining which of these best accounts for changing patterns of illness and death is an important step in understanding both the public health and economic impacts of health intervention in an ageing population. The aim of this study was to evaluate the compression, expansion and dynamic equilibrium theories in Western Australia.

Methods Life tables and survival curves for first-time hospital episodes for chronic disabling and activity limiting conditions and all cause mortality in persons aged 15 or more years in WA in 1980-2003 were constructed using data from the WA Data Linkage System. Changes in life expectancy, average age at first-time hospitalisation and time spent in chronic disabling or activity limiting states were used to evaluate the competing hypotheses.

Results Life expectancy increased by 4.0 and 2.6 years over the 24 year study period in males and females respectively. However, average time spent with a diagnosed chronic disabling condition increased by 8.2 and 8.1 years in males and females respectively, while time spent in an activity limiting state remained largely unchanged.

Conclusion We found evidence to support an expansion of morbidity and some evidence against the dynamic equilibrium theory. This is consistent with population trends towards higher levels of selfreported ill-health in Australia and portends further challenges for the containment of healthcare costs in the future.

\subsection{EXPLORING RISK FACTORS}

\section{Chair: Prof. Harry Campbell, UK 05-6.1 IMMUNOLOGICAL RESPONSE TO HEPATITIS B VACCINATION IN HIV INFECTED PATIENTS: ASSOCIATED FACTORS}

doi:10.1136/jech.2011.142976b.64

G Mena, * A Llupià, A García-Basterio, C Díez, F García, J M Bayas. Hospital Clínic of Barcelona, Barcelona, Spain

Background HIV patients are at a higher risk of hepatitis B virus (HBV) infection than general population. The aim of this study is to assess the immunogenicity of inactivated hepatitis $B$ vaccine among HIV infected patients

Methods A total of $536 \mathrm{HIV}$ infected patients from a university hospital in Barcelona who were vaccinated against HBV between 1996 and 2009 were analysed. Anti-HBs titles were evaluated after the last hepatitis B dose for each patient. Multivariate logistic regression was conducted to identify independent factors (sex, age, hepatitis C coinfection, HIV viral load, CD4 count, type of schedule, dose and number of vaccines) associated with vaccine response.

Results About $71 \%$ of the vaccinated HIV population were respondents (Anti-HBs antibodies $\geq 10 \mathrm{IU} / \mathrm{l}$ ). Vaccine recipients receiving $<3$ doses (OR $0.29,95 \%$ CI 0.16 to 0.52 ) and performing an accelerated schedule (0, 7, 21 days and booster dose at month 6) (OR 2.10, 95\% CI 1.02 to 3.22) were factors associated with a decreased likelihood of a positive response. Development of anti-HBs was higher in patients with CD4 count $\geq 350$ cells $/ \mu l$ (OR 1.78, 95\% CI 1.37 to 2.94), HIV-RNA <50 copies/ml (OR 2.13, 95\% CI 1.41 to 3.22 ) and $40 \mu \mathrm{g}$ dose schedule (OR 1.82, 95\% CI 1.14 to 2.94 )

Conclusions These findings show that a $40 \mu \mathrm{g}$ dose schedule and earlier vaccination from HIV diagnose may be effective strategies for an increased HBV response among HIV infected patients. Anti-HBs titles should be assessed after an accelerated vaccination schedule and after any schedule in patients who present a viral load $<50$ copies/ml or CD4 count $<350$ cells/ $\mu$ l, given the low likelihood of response.

\section{5-6.2 SURGICAL TREATMENT OF ELDERLY BREAST CANCER PATIENTS WITH DISTANT METASTASES AT DIAGNOSIS}

doi:10.1136/jech.2011.142976b.65

${ }^{1}$ E Bastiaannet, ${ }^{*} \mathrm{~S}$ van de Velde, ${ }^{1} \mathrm{~W}$ van de Water, ${ }^{2} \mathrm{M}$ Ernst, ${ }^{3} \mathrm{~A}$ Voogd, ${ }^{1} \mathrm{C}$ van der Velde, ${ }^{1} \mathrm{G}$ J Liefers. 'LUMC, Leiden, The Netherlands; ' ${ }^{2}$ Jeroen Bosch Hospital, Den Bosch, The Netherlands; ${ }^{3}$ CCCS, Eindhoven, The Netherlands

Introduction Several recent retrospective studies have shown a survival gain for patients who received local surgery for metastatic breast cancer. However, data for elderly are not available; so the aim of this study was to assess survival differences according to local surgery for elderly patients (65 years and older) with metastatic breast cancer.

Methods All adult females diagnosed with metastatic breast cancer between 1990 and 2007 were selected from the Netherlands Cancer Registry. Relative survival was calculated as the ratio of survival observed and expected survival based on the corresponding (age, sex 